\title{
1
}

\section{ET: A Third Generation Observatory}

\subsection{Introduction to the third generation of GW observatories}

As described in the previous chapters and according to the current models of GW sources, the advanced interferometric GW detectors (or "second" generation of GW interferometers, like "Advanced LIGO" and "Advanced Virgo") promise the detection of the GW in the first year of operation close to the target sensitivity. For example, at the nominal sensitivity of these apparatuses, a few tens of detected coalescing neutron stars events are expected per year. But, apart from extremely rare events, the expected signal-to-noise ratio (SNR) of these events, in the advanced detectors, is too low for precise astronomical studies of the GW sources and for complementing optical and X-ray observations in the study of fundamental systems and processes in the Universe.

These evaluations and the need of observational precision GW astronomy, led the GW community to start a long investigation process about the future evolution of the advanced detectors to a new ("third") generation of apparatuses (Punturo et al., 2009), with a considerably improved sensitivity. To realize this third generation of GW observatories, having a target of roughly a factor of ten improvement in sensitivity, in a wide frequency range, with respect to the advanced detectors, several limitations of the technologies prepared for the second generation interferometers must be overcome and new solutions must be developed to reduce the fundamental and technical noises that will limit the next generation machines. In effect, the jump from the second to the third generation of GW interferometers is expected to be wider than the step made from the initial to the advanced detectors. Whereas in the $1^{\text {st }} \rightarrow 2^{\text {nd }}$ generation evolution, the technologies adopted in the initial machines are essen- 
tially updated through, e.g. an increase of the injected laser power, an improved seismic noise filtering (in "Advanced LIGO"), a reduction of the low frequency thermal noise by improving the suspension design and suspension fiber material, by adopting the monolithic fused silica design pioneered in GEO600, an improvement of the mirror thermal noise by selecting a better substrate material and coating realization (done in the intermediate step of the enhanced detectors, like e-LIGO and Virgo+), the transition $2^{\text {nd }} \rightarrow 3^{\text {rd }}$ generation will require a complete redesign of the observatory, starting from the infrastructure and the site hosting the interferometers.

In the next sections of this chapter, a subset of the scientific goals of a third generation GW detector and a short overview of the technological challenges introduced by this new generation of machines, currently under evaluation within the framework of the Einstein Telescope (ET) design study (http://www.et-gw.eu/), will be described. It is worth to cite here that, inside the ET scientific community, it has been suggested to use the word "observatory" rather than "detector" when referring to the third generation of interferometers, to underline the fact that the main component (costwise) of these apparatuses will be the infra-structure, which will last for decades and will be made compatible with subsequent upgrades of the hosted detectors, and the main scientific target will be the observation of the GW sources, rather than their detection, which is expected to happen with the advanced detectors.

\subsection{Science potential of a $3^{\text {rd }}$ generation GW observatory}

The definition of the expected sensitivity of a third generation GW observatory is a trade off between the science targets, the technological achievements and the financial costs of the project. In the Einstein Telescope design study (http://www.et-gw.eu/) an improvement in sensitivity by a factor of 10 , with respect to the advanced detectors, and the possibility to access the $1-10 \mathrm{~Hz}$ frequency range, still inaccessible for the $2^{\text {nd }}$ generation, has been indicated as a target. This resulted in a possible target sensitivity (named "ET-B"), shown in Fig. 1.1.

Such a sensitivity will open a new window for understanding the physics of extreme phenomena in the universe; hereafter a short list of possible scientific targets, discussed in more detail in the ET science vision document (Amaro-Seoane et al., 2009), is described: 


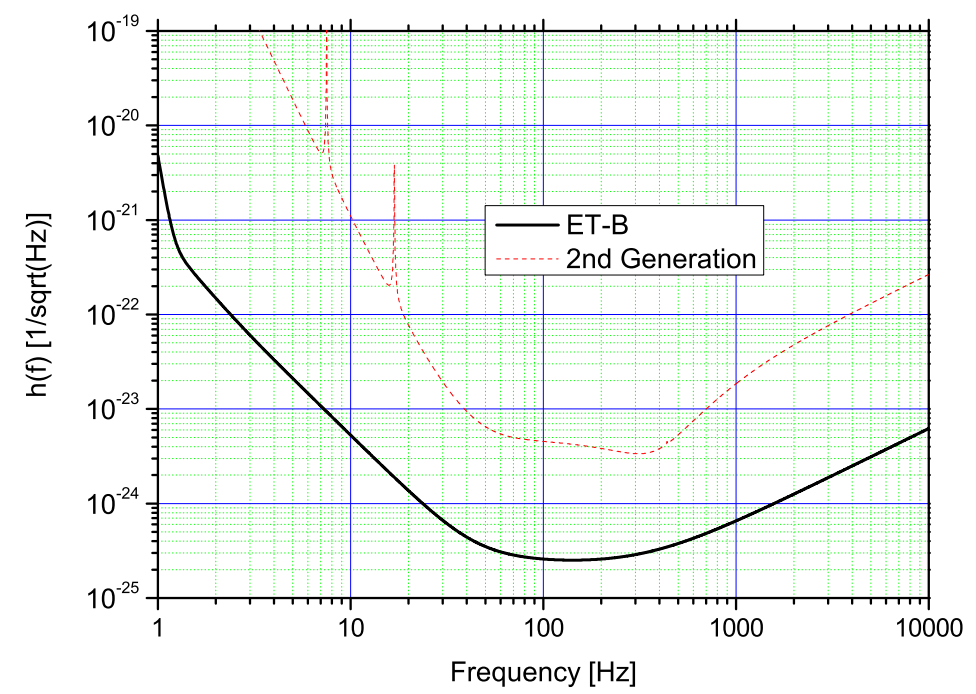

Figure 1.1 A possible sensitivity (solid curve) of an underground, long suspension, cryogenic, signal and power recycled single third generation gravitational wave observatory (see Table 1 in (Hild et al. (2008))) compared to a typical sensitivity curve of an advanced detector (dashed curve). It is worth to underline that the evaluation of the possible noise level of a third generation GW observatory is an ongoing activity, still far to be concluded within the ET design study. For this reason the curves are updated regularly and labeled with progressive letters to be distinguished. In the solid curve (so-called ET-B), corresponding to a single wide-band detector, the suspension thermal noise contribution is missing.

Astrophysics: Measure in great detail the physical parameters of compact stars [i.e., neutron stars (NS) and black holes (BH)] in a binary system (Broeck, 2006; Broeck and Sengupta, 2007), constrain the equation-of-state of NS and solve the enigma of gamma ray bursts (GRB) (Nakar, 2007; Amaro-Seoane et al., 2009).

General Relativity: Test general relativity by comparing observations of massive binary star systems with numerical relativity (NR) predictions and constrain alternative theories of gravity (such 
as the Brans-Dicke theory) through the observation of NS-BH coalescences (Amaro-Seoane et al., 2009).

Cosmology: Measure cosmological parameters from standard sirens of gravity (Schutz, 1986; Sathyaprakash et al., 2009) and probe the primordial Universe through the measurement of the GW stochastic background (Amaro-Seoane et al., 2009).

Astroparticle physics: Measure or constrain the neutrino (Arnaud et al., 2002) and graviton masses through the detection of the GW emitted in a supernova.

\subsubsection{Binary systems}

Binary systems of neutron stars (BNS) or black holes $(\mathrm{BH}-\mathrm{BH})$ are the main targets of the advanced GW detectors; the expected rate of BNS coalescence events within the sight distance of $\lesssim 400 \mathrm{Mpc}$ is of the order of few tens per year and, hence, the detection should be guaranteed, although the low signal-to-noise ratio (SNR $\gtrsim 8)$ will obstruct a detailed measurement of the physics properties of the GW signal sources. The enhanced sensitivity of the third generation GW observatories, instead, will permit a detailed measurement of the characteristics of the GW source with a relevant impact, for example, on cosmography. BNS are considered standard sirens (Schutz, 1986; Sathyaprakash et al., 2009); in fact, by measuring the GW signal with a network of (at least) three detectors, it is possible to evaluate the chirp mass $M$ (because the chirp rate depends on $\mathrm{M}$ ) and the amplitude, allowing a direct evaluation of the BNS luminosity distance $D_{L}$. GW cannot, though, determine the red-shift $z$ of a source (and an ambiguity between intrinsic and observed total BNS mass remains) and hence an electro-magnetic counterpart is needed. In Fig. 1.2, upper panel, the BNS detection range for the ET$\mathrm{B}$ sensitivity is shown, revealing a sight distance of about $z \sim 2$ and a impressive SNR at large distances (as shown in Fig. 1.2, lower panel, at $3 \mathrm{Gpc}$ a SNR of several tens is possible). With this performance, about $10^{6} \mathrm{BNS}$ coalescences per year are expected in the ET detection range; let's suppose that in about $1 / 1000$ of these events the red-shift could be measured identifying them with coinciding gamma-ray bursts (GRB). Luminosity distance $D_{L}$ and the corresponding red-shift $z$ are related by the adopted cosmological model; by introducing these two measured quantities in equation 1.1, describing the model, it is possible the evaluate some of the fundamental cosmological parameters, like the total mass density $\Omega_{M}$, the dark energy density $\Omega_{\Lambda}$ and the dark energy 
equation of state parameter $w$ ( $H_{0}$ in Eq.1.1, is the Hubble parameter).

$$
D_{L}=\frac{c(1+z)}{H_{0}} \int_{0}^{z} \frac{d z^{\prime}}{\left[\Omega_{M}\left(1+z^{\prime}\right)^{3}+\Omega_{\Lambda}\left(1+z^{\prime}\right)^{3(1+w)}\right]^{1 / 2}}
$$

The expected errors in the evaluation of these cosmological parameters, computed in (Sathyaprakash et al., 2009) are listed in Table 1.1 and are comparable to those from other cosmological measurement attempts, such as the Joint Dark Energy Mission.

Table 1.1 Errors in the determination of the cosmological parameters through the detection of 5192 realizations of a catalogue containing 1000 BNS merger events, of known red-shift (Sathyaprakash et al., 2009). The fractional 1- $\sigma$ width of the distributions $\sigma_{\Omega_{\Lambda}} / \Omega_{\Lambda}, \sigma_{\Omega_{M}} / \Omega_{M}$ and $\sigma_{w} /|w|$ are shown, accounting for the weak lensing errors in the left column and considering it corrected in the right column. Briefly:

$\Omega_{M}$ and $\Omega_{\Lambda}$ are the (dimensionless) energy densities of the Dark

Matter and Dark Energy, respectively; $w$ is the Dark Energy equation of state parameter ( $w=1$ corresponds to a cosmological constant).

\begin{tabular}{ccccccc}
\hline Free parameters & \multicolumn{2}{c}{$\sigma_{\Omega_{\Lambda}} / \Omega_{\Lambda}$} & \multicolumn{2}{c}{$\sigma_{\Omega_{M}} / \Omega_{M}$} & \multicolumn{2}{c}{$\sigma_{w} /|w|$} \\
\hline 3 & $4.2 \%$ & $3.5 \%$ & $18 \%$ & $14 \%$ & $18 \%$ & $15 \%$ \\
\hline 2 & $\Omega_{\Lambda}=0.73$ & $9.4 \%$ & $8.1 \%$ & $7.6 \%$ & $6.6 \%$ \\
\hline 1 & $\Omega_{\Lambda}=0.73$ & $\Omega_{M}=0.27$ & $1.4 \%$ & $1.1 \%$ \\
\hline
\end{tabular}

The detection distance and the SNR shown in Fig. 1.2 are computed using templates produced in the so-called restricted post-Newtonian $(\mathrm{PN})$ approximation, where only the lowest order (corresponding to the second harmonic of the orbital frequency) of the PN expansion is retained. Although the potential of the sub-dominant higher harmonics aren't fully evaluated, it is clear (Broeck, 2006; Broeck and Sengupta, 2007; Arun et al., 2007) that they could facilitate the observation of heavier binary systems (by enriching the high frequency part of the GW spectrum) as well as greatly improve the accuracy in the measurement of the GW source parameters (relaxing, also, the requirement on the minimal number of detectors in the network needed to resolve a GW source). All this will allow the determination of the mass function of neutron stars and black holes, the maximum mass of a neutron star and its equation-of-state and provide a comprehensive history of the formation and evolution of compact binaries. As described in (Gair et al., 
2009a,b), such studies will give answers to important astrophysical questions such as the history of star formation, the birth of intermediate-mass black holes and their growth, etc.

When the two massive stars (NS or better $\mathrm{BH}$ ) in a binary system are close to the final coalescence, because of the ultra strong gravitational fields, the PN approximation is no longer valid and a fully relativistic treatment is needed. Thanks to the advances in analytical (Buonanno and Damour, 1999, 2000; Damour et al., 2002, 2003; Damour and Nagar, 2007b,a; Buonanno et al., 2007) and numerical relativity (Brügmann et al., 2004; Pretorius, 2005; Baker et al., 2007; Boyle et al., 2007; Ajith et al., 2008; Hannam and Hawke, 2009) it is possible to predict the characteristics of the signal emitted in the process of merger. Comparison of the predicted signal amplitudes and fluxes with the observation carried on by ET will enable new tests of general relativity. It should be possible to check whether the spacetime geometry of the merged object is that of a black hole or some other exotic object, whether black holes are enclosed in a horizon, etc.

\subsubsection{Isolated Neutron Stars}

As explained in the previous chapters, Neutron stars (NS) are one possible end state of massive stars that result in a gravitational collapse followed by a supernova of Type II, Type Ib or Type Ic. The composition of a NS is still under debate and the measurement of the GW emitted by such as body could give important information on its internal structure. In particular, being the main mechanism for the emission of GWs by a NS due to the quadrupolar moment generated by its ellipticity $\epsilon$, from its measurement it is possible to deduce important information on the equation-of-state of the ultra-dense matter composing the NS. Its is worth to underline that, according to current NS models, a solid core NS could sustain $\epsilon \lesssim 10^{-3}$, whereas the crust of the NS could support up to $\epsilon \simeq 10^{-6}-10^{-7}$. Because of its improved performance, a third generation GW observatory could be sensitive to the GW emitted by isolated NS having a relatively small ellipticity, as shown in Fig. 1.3 and permits to cover a large fraction of our galaxy in a blind search (see Fig. 1.4). 


\subsubsection{Supernova explosion}

In a supernova explosion ( $\mathrm{SNe}$ ) caused by the collapse of the core of a NS the energy radiated in GWs is expected to be a very small fraction of the total energy emitted (still under debate, but less than $10^{-7}-10^{-8}$ solar masses, according to what is reported in the review (Ott, 2009)). This fact limits the detection potential of the initial and advanced detectors to the Milky Way and the closest galaxies, with an expected detection rate of about 1 event every 2 decades in the Local Group (Bergh and Tammann, 1991). ET could detect SNe up to few Mpc, with an expected SNe rate of 1 event every 1-2 years (Ando et al., 2005) (if we consider a detection distance of $5-6 \mathrm{Mpc}$ ). If, as expected, in the timescale of the implementation of the third generation of GW observatories, Megaton-class neutrino detectors are operational and have a detection range comparable to ET (as indicated in (Ando et al., 2005)), it will be possible to provide coincident observation with non-negligible detection rate, permitting important evaluations on the SNe mechanisms and on the neutrino mass value.

\subsection{Third generation sensitivity: how to suppress the noises limiting the advanced GW detectors}

In Fig. 1.1 a possible target sensitivity (so-called ET-B) of a third generation GW observatory is compared with the expected sensitivity of an advanced detector. To bridge the gap between the two curves, several noise sources must be suppressed. Obviously a conceptually easy way to improve the sensitivity is to realize a new interferometer with longer arms and, indeed, the ET-B curve is computed assuming an arm length of $10 \mathrm{~km}$; this is the first trivial indicator of the need of a new infrastructure for the third generation. But, to act on the several noises limiting the nominal sensitivity of the advanced detectors, in different frequency ranges, new and specific technological solutions must be found. At very low frequencies (below $3-4 \mathrm{~Hz}$ ) the seismic and the related gravity gradient noises must be suppressed. In the $4-50 \mathrm{~Hz}$ range the thermal noise of the optics suspension system and the quantum noise, related to the radiation pressure exerted on the suspended mirror by the photons in the main Fabry-Perot cavities must be reduced. Above $40 \mathrm{~Hz}$, the thermal noise (dominated by the mechanical dissipation of the high reflectivity dielectric coating) of the suspended mirror and the shot noise compo- 
nent of the quantum noise play a dominant role and must be reduced simultaneously to achieve the target sensitivity. The following subsections are devoted to the description of the limiting noises and to the possible technological solution in a third generation GW observatory.

\subsubsection{Seismic and Gravity Gradient noise}

At low frequency, ground based interferometric GW detectors are limited by the natural and anthropogenic vibration of the ground where the apparatus is built. Seismic excitation acts on the suspended test masses both indirectly, through the suspension chain, shaking each stage according to its transfer function, and directly, coupling the mass vibration in the soil layers, perturbed by the seismic waves, with the test-mass displacement, via the mutual attraction force expressed by Newton's universal law of gravitation (so-called Gravity Gradient noise or Newtonian noise).

Advanced detectors will implement seismic filtering systems to reduce the test-mass shaking due to the ground vibration. "Advanced LIGO" will implement a so-called active filtering system (Abbott et al., 2002) where, in a chain of three sub-systems, the displacements and the accelerations caused by the seismic noise are read through position and acceleration sensors and are actively and hierarchically suppressed through hydraulic and electro-magnetic actuators. "Advanced Virgo" will adopt, instead, the passive filtering philosophy already successfully implemented in the initial Virgo, based on a chain of harmonic oscillators filtering the seismic vibration horizontally (inverted and "normal" pendulums) and vertically (blades). The so-called Virgo Super-Attenuator pushes the residual seismic noise below the thermal noise of a first generation detector like Virgo starting from about $4 \mathrm{~Hz}$ (Braccini et al., 2005) and it has been demonstrated (Braccini, 2009) the compliance of this apparatus with "Advanced Virgo".

In the ET design, there are additional requirements that make the achievement of the sensitivity target very challenging; in fact, beyond the request of reducing the noise level, roughly by a factor of ten, in the whole detection frequency band, the requirement to access the frequency region between $1 \mathrm{~Hz}$ and $10 \mathrm{~Hz}$, excluded in the advanced detectors, is too difficult to be obtained just with an improved seismic filtering system and alternative (or better additional) solutions must be found. It is well known that underground sites are seismically quieter (i.e. see (Beker et al., 2009)) and the possibility to realise an underground GW detector 
has been analysed and selected by the LCGT collaboration in Japan. The comparison between the seismic noise in the TAMA site (Tokyo) and in the LISM (Sato et al., 2004) site (Kamioka mine, the prime candidate for an LCGT site) shows a reduction in the low-frequency region, by going underground, by a factor of 100 in terms of acceleration and by two to three orders of magnitude in displacement spectral amplitude. A corresponding and even larger noise reduction has been reached in the output of the LISM interferometer, due to the fact that going underground several other "technical" noises, induced by external disturbances like wind, scattered light or temperature fluctuations, are suppressed by the quietness of the site. Hence, to achieve the ET-B target sensitivity an underground site is mandatory; for these conditions, it has been shown (Braccini, 2009) that the Virgo Super-Attenuator satisfies the ET requirements above $3-4 \mathrm{~Hz}$, whereas to access the $1-3 \mathrm{~Hz}$ frequency range major technical upgrades of the suspension system have to be realised. This is the second, more important, indicator that a new infrastructure is a priority issue on the path towards the realization of the third generation of GW observatories.

The Gravity Gradient noise effect has been modelled for an interferometer on the Earth's surface (Saulson, 1984; Beccaria et al., 1998; Hughes and Thorne, 1998) and the predicted noise level is negligible for the initial and advanced detectors. In a third generation GW detector, the more stringent requirements at low frequencies, accentuate the role of this noise source. It has been shown (Cella, 2009) that, going deeply underground ( $\gtrsim 100 \mathrm{~m}$ ) it is possible to effectively suppress the gravity gradient noise (at least above $2 \mathrm{~Hz}$ ). Further reductions require a major effort to complement the seismic attenuation with the subtraction of the residual gravity gradient noise through signals extracted from a network of sensors located around the detector (Cella, 2009).

\subsubsection{Thermal Noise}

As previously cited, the so-called thermal noise, generated by all the processes modulating the optical path of the light in the interferometer, coupling it to the Brownian fluctuation or to the stochastic fluctuation of the temperature field in the optical components, dominates the sensitivity of the initial and advanced detectors in the low and medium frequency range $(4-200 \mathrm{~Hz})$. To model and understand the thermal noise in the interferometers (in thermal equilibrium) two fundamental instruments are used: the equi-partition theorem, that relates the temperature 
of a system to its average energies, and the Fluctuation-Dissipation (FD) theorem (Callen and Welton, 1951), that relates the power spectrum of the fluctuations of a system in thermal equilibrium to the dissipation processes, described by its mechanical impedance.

In the initial and advanced interferometers, the unique tool used to reduce the thermal noise effect on the detector has been the reduction of the dissipation processes through the selection of the best material available (i.e. C85 steel in Virgo (Cagnoli et al., 1999) or fused silica in GEO600 (Goßler et al., 2004) and the advanced detectors (Amico et al., 2002; Cagnoli et al., 2006)) or trough the minimization of the clamping losses (i.e. by optimizing the clamping design (Cagnoli et al., 1996) in initial detectors, or by introducing the monolithic design in GEO600 and advanced detectors).

In the third generation of GW observatories a further reduction of the thermal noise limitation must be found and, in the ET design, as well as in the LCGT project, the additional methodology is based on the reduction of the interferometer operational temperature down to a few degree Kelvin. In fact, according to the equi-partition theorem, temperature is directly proportional to the energy stored in each degree of freedom of the suspended system allowing to reduce the fluctuation amplitude by lowering the suspension temperature. Furthermore, at low temperature, some materials show a suppression of the dissipation mechanisms.

It is really important to select the right technology to cool down the suspended mirrors of the interferometer without introducing additional noise (like seismic and acoustic excitation caused by the cryogenic liquid boiling processes, or the mechanical pumps vibration). Cryo-coolings systems are really appealing because they promise an excellent duty cycle to the interferometer, hence, cryogenics is one of the most appealing technologies to reduce the thermal noise of the optics suspension in a third generation gravitational wave detector. The first problem to be solved in a cryo-interferometer is how to cool down the test masses without introducing additional vibrations that spoils the very low frequency performances. A promising technology is now available, based on cryo-cooling systems, dampened to reduce the seismic vibration (Tomaru et al., 2004).

The cryogenic solution introduces a discontinuity in the evolution of the materials adopted in GW interferometers. In fact, the monolithic suspension, developed for the second generation of gravitational wave detectors, cannot be used in cryogenics because of the poor thermal conductivity of the fused silica and because of a well known dissipation 
peak of that amorphous material at low temperature. The requirements that a material should satisfy, to be electible for a cryogenic suspension, are a high thermal conductivity at the operation temperature, to permit an efficient heat extraction (which is crucial, because of the relatively large heating power deposited in the test masses by the high light power stored in the interferometer cavities), a low mechanical dissipation angle (to reduce the Brownian thermal noise), a low thermal expansion coefficient (to minimise the thermo-elastic noise), and a good breaking strength (to safely support the test masses). Currently there are two candidate materials for this role: Sapphire and Silicon. Sapphire has been selected to realise the suspension fibres of LCGT both for its dissipation (Uchiyama et al., 2000) and for its thermal conductivity properties (Tomaru et al., 2002); also Silicon has been found a suitable material to realise both suspension fibres (Alshourbagy et al., 2006) and ribbons (Reid et al., 2006). However, as matter of fact currently only Sapphire has been used to realize a full cryogenic suspension and the usage of Silicon still needs a successful R \& D activity.

At intermediate frequency $(40-200 \mathrm{~Hz})$ it is the thermal noise, related to the losses in the suspended test masses, that dominates the noise budget of the advanced detectors. To be more precise, the mechanical dissipation located in the high reflectivity dielectric coatings (mainly in the Tantalum-pentoxide $\mathrm{Ta}_{2} \mathrm{O}_{5}$ layers) gives the largest contribution to the noise level in that frequency range. A large R\&D effort in the realization of the advanced detectors is devoted to reduce that effect both by decreasing the dissipation of the $\mathrm{Ta}_{2} \mathrm{O}_{5}$ layers, introducing a Titanium dopant (Harry et al., 2007), and by reducing the total amount of Tantalum-pentoxide material while keeping the same reflectivity (Agresti et al., 2006). These developments are important also for the third generation of GW observatories, but the low temperature requirement introduces additional problems. In fact, it has been shown that going at low temperature, in a multi-layer Tantalum-pentoxide coating, the mechanical dissipation is rather constant (Yamamoto et al., 2006) and more recent measurements (Martin et al., 2008) have even shown a low temperature dissipation peak in a single-layer of $\mathrm{Ta}_{2} \mathrm{O}_{5}$ doped with $\mathrm{TiO}_{2}$. Furthermore, because of the previously mentioned broad dissipation peak shown by fused silica at low temperature, in the cryogenic interferometers it is impossible to choose the low mechanical loss, low optical absorption substrates developed for the advanced detectors. The best material candidate to realize the test masses is, again, Sapphire (selected in LCGT) and crystalline Silicon, which shows a very 
low mechanical dissipation angle (about $3-4 \times 10^{-9}$ ) at low temperature. The use of a Sapphire test masses simplifies the development of all the electro-optical components of a third generation GW observatory, since it is transparent to the standard wavelength adopted in the gravitational wave detectors $(1064 \mathrm{~nm})$. Furthermore, it shows relatively small thermal lensing (Tomaru et al., 2002) due to its large thermal conductivity at low temperature $\left(2330 \mathrm{~W} \cdot \mathrm{m}^{-1} \mathrm{~K}^{-1}\right.$ at $\left.10 \mathrm{~K}\right)$, but the high optical absorption (about $90 \mathrm{ppm} / \mathrm{cm}$ (Tomaru et al., 2001)) measured in the available substrate samples, constrains the interferometer design and limits the future light power increase in the main Fabry-Perot cavities. Obviously, future improvements in the optical properties of Sapphire substrates could enhance the possibilities of this solution. Silicon shows a similar thermal conductivity $\left(1200 \mathrm{~W} \cdot \mathrm{m}^{-1} \mathrm{~K}^{-1}\right.$ at $\left.12.5 \mathrm{~K}\right)$, but it is transparent only at a longer wavelength $(\lambda \gtrsim 1450 \mathrm{~nm})$, where it shows a very low absorption (about $3 \times 10^{-8} \mathrm{~cm}^{-1}$ at $1445 \mathrm{~nm}$ (Green and Keevers, 1995)), which requires to reconsider all optical and electro-optical component choices in the interferometer.

A solution of this problem is searched both by investigating possible new high refraction index materials, that must show a low dissipation at cryogenic temperature when used in a dielectric coating, and by looking for a complete different approaches. In this second scenario, an arbitrary selection of interesting R \& D activities is hereafter briefly described. The first promising possibility (Brückner et al., 2009) is related to the production of a high reflectivity mirrors with just one dissipative layer of dielectric coating material on the substrate or even without any additional layer, realizing the so-called resonant waveguide grating (Bunkowski et al., 2006) by nano-structuring the surface of the Silicon substrate. Another possible innovative solution could be based upon the so-called "Khalili cavities" (Khalili, 2005), that permit to build high finesse Fabry-Perot cavities minimizing the thickness of the dissipative high reflectivity coating layers, by replacing the end mirrors with additional anti-resonant cavities. This option is currently preliminary being investigated in ET (Hild, 2009) and an overall reduction factor of the coating Brownian thermal noise of 1.5 has been evaluated; the realization difficulties (thermal load on the end mirror substrates, short end cavity control issues, etc.) are still to be investigated. Finally, a further method, under investigation in ET, that promises an effective reduction of the thermal noise level, is the use of flatter beams in the Fabry-Perot cavities. In fact, it is well known that the effect of the mirror thermal noise fluctuation on the detector sensitivity diminishes with the beam 
size in the cavity, but, because of the physical limitation on the mirror substrate size and on the acceptable level of diffraction losses, it is difficult to have beam sizes in ET that are substantially larger than the beams in the advanced detectors. For this reason, the possibility to use a beam with an intensity distribution flatter than the Gaussian one is appealing and the thermal noise reduction using higher-order LaguerreGauss modes has been investigated (Chelkowski et al., 2009).

\subsubsection{Quantum Noise}

As could be seen in the chapters discussing the second generation of GW detectors, i.e. the 'advanced' instruments, Quantum Noises are important both a the high and the low frequency end of the detection band. At high frequencies shot noise typically dominates the sensivity, whereas the importance of radiation pressure noise increases towards lower frequencies. Both forms of Quantum Noise need to be lowered in order to reach the sensitivity goals for a third generation observatory.

Shot noise is an apparent test mass displacement noise as it creates a signal but no real mirror motion. Differential phase noise on the laser beams in the interferometer arms gets converted into amplitude fluctuations of the recombined beam in the output port and hence cause measurable noise on the photo detector. There are two ways of improving the shot noise: increasing the signal and lowering the noise.

The easiest way to increase the signal is increasing the light power in the interferometer arms. The signal size obtained from the photo detector at the output port created by a GW of a given amplitude linearly depends on the light power, whereas the shot noise scales as the square root of the light power. Hence the Signal-to-Noise Ratio (SNR) also scales as the square root of the light power.

In the Einstein Telescope a light power in the interferometer arms of a few MW may be necessary. This can be achieved with a high power laser of about $1 \mathrm{~kW}$ power, arm cavities and Power Recycling as a straight forward extension of current technologies. The problems arising from increased local heating by absorbed laser power will require sophisticated thermal compensation schemes, similar to the ones used for the first generation of GW detectors (Waldman for the LIGO Science Collaboration (2006) and Acernese et al. (2008)). Like in the second generation of detectors, Signal Recycling (Meers (1988)) can be used to further increase the signal in parts of the frequency band.

The use of high light power in the interferometer arms can lead to an- 
other problem: parametric instabilities (Braginsky et al. (2001)). Parametric instabilities arise from a coupling of higher order modes in resonant optical cavities and acoustic modes of the cavity mirror substrates and can cause serious problems for a stable operation of the advanced interferometers (Ju et al. (2006b) and Ju et al. (2006a)). Studies for LCGT (Yamamoto et al. (2008)) have shown that the problem is less severe in this interferometer because of the different geometry and materials of the mirrors, since the radius of curvature of the mirror and the sound velocity at the operative temperature play a relevant role in determining the number of instable modes. Parametric instabilities for the Einstein Telescope have been investigated showing that the number of instable modes is much bigger than in LCGT (Yamamoto et al. (2008)) and most likely need suppression techniques. A reduction of the mechanical Q of the elastic mirror modes in a way that does not compromise the thermal noise to an intolerable extent is a promising technique for treating the parametric instabilities. Active feedback with external actuators, e.g. radiation pressure actuators, is also possible but requires sophisticated control loops to safely identify and suppress all possible instable modes.

The sensitivity of the interferometer can not only be improved by increasing the signal but also by lowering the quantum noise. Lowering the shot noise requires lowering the differential phase fluctuations in the interferometer arms. This can be achieved by injecting squeezed vacuum states into the output port of the interferometer. For details we would like to refer to Kimble et al. (2001) and Harms et al. (2003). Squeezed states trade the fluctuations in one quadrature of the field against fluctuation in the other one. Lowering the phase fluctuations will inevitably increase the amplitude fluctuations, as both quadratures are related to each other by Heisenberg's uncertainty relation. Hence if the noise in the squeezed light is lowered in the quadrature that coincides with the carrier amplitude at the output port in case of DC readout (often called phase quadrature as it causes differential phase noise in the interferometer arms) the shot noise gets lowered but the amplitude noise in the interferometer arms gets increased. At present squeezing levels of more than 10dB can be reached (Vahlbruch et al. (2008) and Mehmet et al. (2009)) and the squeezing can cover the full frequency range aimed for (Vahlbruch et al. (2006)). For the time of operation of the Einstein Telescope we anticipate the sum of losses in the path from the squeezing source via the interferometer to the photo detector to be low enough (i.e. on the order of 5\%) and squeezing levels to be high enough (about $15 \mathrm{~dB}$ ) to obtain an effective squeezing of $10 \mathrm{~dB}$, i.e. a 
factor of 10 in power spectral density, corresponding to the same SNR improvement as a light power increase of a factor of 10 .

Noise in the amplitude quadrature of the light in the interferometer arms causes physical motion of the test masses by the momentum transfer of the light being reflected. This noise is called radiation pressure noise and rises with the inverse of the square of the signal frequency, as the mechanical susceptibility due to inertia rises towards lower frequencies. The radiation pressure effect can be lowered by increasing the mirror mass. Mirror masses on the order of $500 \mathrm{~kg}$ are considered for the Einstein Telescope. In the same way as the shot noise can be improved with squeezed light in one quadrature, radiation pressure noise can be lowered by squeezing the other quadrature of light being injected into the output port. In order to gain from squeezing in shot noise and radiation pressure noise, different quadratures will have to be used for the respective frequency ranges. This can be achieved by using filtering cavities (Kimble et al. (2001)). The optimal way of filtering the light before detection and filtering the squeezed state before injection for different interferometer topologies for the Einstein Telescope is being investigated.

\subsubsection{Scenarios for the third generation}

The realization of a third generation GW observatory could follow different paths according to the chosen scientific targets, to the selected technologies and to the financial investment to the project. In the following sections a possible scenario is depicted, according to the studies performed within the ET design study.

\section{Single detector or multi-detector observatory}

As described in the previous sections, a schematic picture of a third generation GW observatory could be based on the following options:

- long arms, probably about $10 \mathrm{~km}$ long, to enhance the sensitivity to the dimensionless space-time strain $h$;

- underground site, to suppress the seismic and gravity gradient noises;

- longer seismic filtering chains, with respect to advanced detectors, to push down the low frequency limit toward $1 \mathrm{~Hz}$;

- cryogenic test masses, to suppress suspension and mirror thermal noises;

- large and flat beams, to suppress thermal noise and mitigate the mirror thermal lensing; 
- high power laser (about $1 \mathrm{~kW}$ ), high finesse Fabry-Perot cavities, high power recycling factor, signal recycling and squeezed light state injection, to suppress the quantum (shot) noise;

- heavy test masses and filtered squeezed state injection, to suppress radiation pressure noise.

All these options have advantages, drawbacks and constraints and, in a realistic design of a $\mathrm{GW}$ detector, the identification of the limitations of each technology and of the cross-incompatibilities is crucial. A first evaluation of the potential of a detector implementing all these options, disregarding the cross-incompatibilities between the selected technologies, has been performed (Hild et al., 2008) within the ET project. Targeting for a wide-band detector, the sensitivity (named ET-B) of an underground, long suspension, cryogenic, signal and power recycled single Fabry-Perot enhanced Michelson detector has been evaluated (see Table 1 in (Hild et al., 2008)) and the resulting sensitivity is plotted in Fig. 1.1. In this evaluation, the cross-compatibility between the different technologies has been neglected, but the technological difficulties are evident. For example, the need of high power in the Fabry-Perot cavities conflicts with the requirement of a cryogenic suspension optimized for thermal noise. In fact, let suppose to realize a coating on the suspended mirrors that shows an excellent absorption of about $0.1 \mathrm{ppm}$; if the power stored in the cavities is about $3 \mathrm{MW}$, the deposited power is $300 \mathrm{~mW}$. To extract that heat from the test mass, although both the Sapphire and Silicon show an excellent thermal conductivity at low temperature, a cross section of several tens of $\mathrm{mm}^{2}$ is needed (corresponding to suspend the test masses with rods of few mm of radius). Obviously this affects the geometrical dilution factor of the suspension, spoiling the pendulum thermal noise, and increases the coupling between the angular and translation degrees of freedom of the suspension, making more complex and, probably, noisy the control of the suspended test mass. Therefore the appealing opportunity to simplify the technological difficulties, merging in a wide-band third generation observatory the output of two (or more) detectors, specialised on different frequency bands, has been evaluated in (Hild et al., 2010). Here the output of a low-frequency-specialised detector is combined with the output of a high-frequency interferometer. The former one could be a cryogenic interferometer at an underground site, with long suspensions, but moderate optical power, whereas the high frequency interferometer could essentially be a long arm advanced detector, implementing squeezed light states, a very high power laser 
and large test masses. The main advantage of this so-called Xylophone philosophy (Shoemaker, 2001; Conforto and DeSalvo, 2004) is the fact that it decouples the technological requirements of a high power interferometer from the requirements of a cryogenic detector, simplifying the realization of an overall wide-band observatory that evades the crossincompatibilities of the implemented technologies; obviously, as additional benefit, the capability to independently tune the sensitivities of the two (or more) detectors constituting the observatory permits to better fit the sensitivity of such an observatory to the searched GW sources. A possible realization of such as Xylophone strategy, evaluated in (Hild et al., 2010) for the ET design study, is plotted in Fig. 1.5.

\section{Detector Geometry}

Because of the quadrupolar nature of the $\mathrm{GW}$, the L-shaped, with orthogonal arms, geometry optimises, with respect to the arm length, the sensitivity of a detector and, consequently, all the currently active $\mathrm{GW}$ detectors are $90^{\circ} \mathrm{L}$-shaped. But other geometries are possible; in particular, triangular-shaped detectors have been proposed in the past (Winkler et al., 1985) and, obviously, also the LISA geometry is triangular. A detailed analysis of the benefits and drawbacks of a triangular-shaped third generation GW observatory is described in (Freise et al., 2009) and here few highlights are reported. In a third generation GW observatory, to fully profit of the site infrastructures, that represent, by far, the dominant part of the costs (underground site excavation, cryogenics, long vacuum pipes), it is probable that more than one wide-band detector is installed.

Co-located interferometers could be extremely useful to extract additional information from the GW observation; for example, two L-shaped detectors, forming a $45^{\circ}$ angle, could fully resolve the two polarisation amplitudes of the incoming wave. Three co-located interferometers, rotated by an arbitrary angle, through the virtual interferometry technique, could do the same, supplying additional benefits like null-stream channels and redundancy. The implementation, in an underground site, of a similar cluster of orthogonal L-shaped detectors presents several drawbacks, mainly because of the large number of tunnels and caverns to be realized to host the detectors and, consequently, the huge cost of the infrastructure. Some optimization of the costs is considered possible if the angle between the two arms of each detector is reduced to $60^{\circ}$ and three detectors can be accommodated in a triangular-shaped underground site, minimizing the total length of tunnels, probably the 
number of caverns and recovering a sensitivity equivalent to two sets of orthogonal L-shaped double detectors, rotated by $45^{\circ}$ (see Fig. B1 of (Freise et al., 2009)).

The selection of the observatory geometry, because of the dominant role of the cost of the infrastructures, will probably be driven by the selection of the site and not vice versa: if a site that can accommodate a triangular observatory is found, the triple co-located interferometers will be the best choice, otherwise the two sets of orthogonal L-shaped double detectors will be more appealing.

\subsubsection{Timelines}

The evolution to the third generation of GW observatories has been, is and will be a long path. Currently the main effort is made in Europe and only the European scenario will be depicted, but it is crucial to have a network of third generation GW observatories in the world. In fact, although the enhanced sensitivity of this generation of interferometers permits to improve the directionality of each observatory, i.e. by using the additional information embedded in the higher harmonics of the full PN modeling of the GW signal, it is recommended to have a network of three distant detectors to reconstruct the position of the GW source.

After a series of preliminary activities supported by the European Commission within the Framework Programme 6 (FP6), a conceptual design study has been funded in the 2008-2011 Framework Programme 7 (FP7): the Einstein Telescope design study (http://www.et-gw.eu/). The main goal of the ET project is to deliver a conceptual design for such a facility, investigating the technological feasibility, the science targets, the site requirements and prepare a costing draft for the infrastructure and a list of site candidates.

Other design and preparation phases will follow the end of the conceptual design and it is expected that, after the first detection of GW in the advanced interferometers, there will be the possibility to start with the realization of the first site of a third generation GW observatory: the Einstein Telescope observatory. In the current plans, it will occur approximately in 2018-2019, followed by several years of construction and commissioning of the detectors.

\section{References}



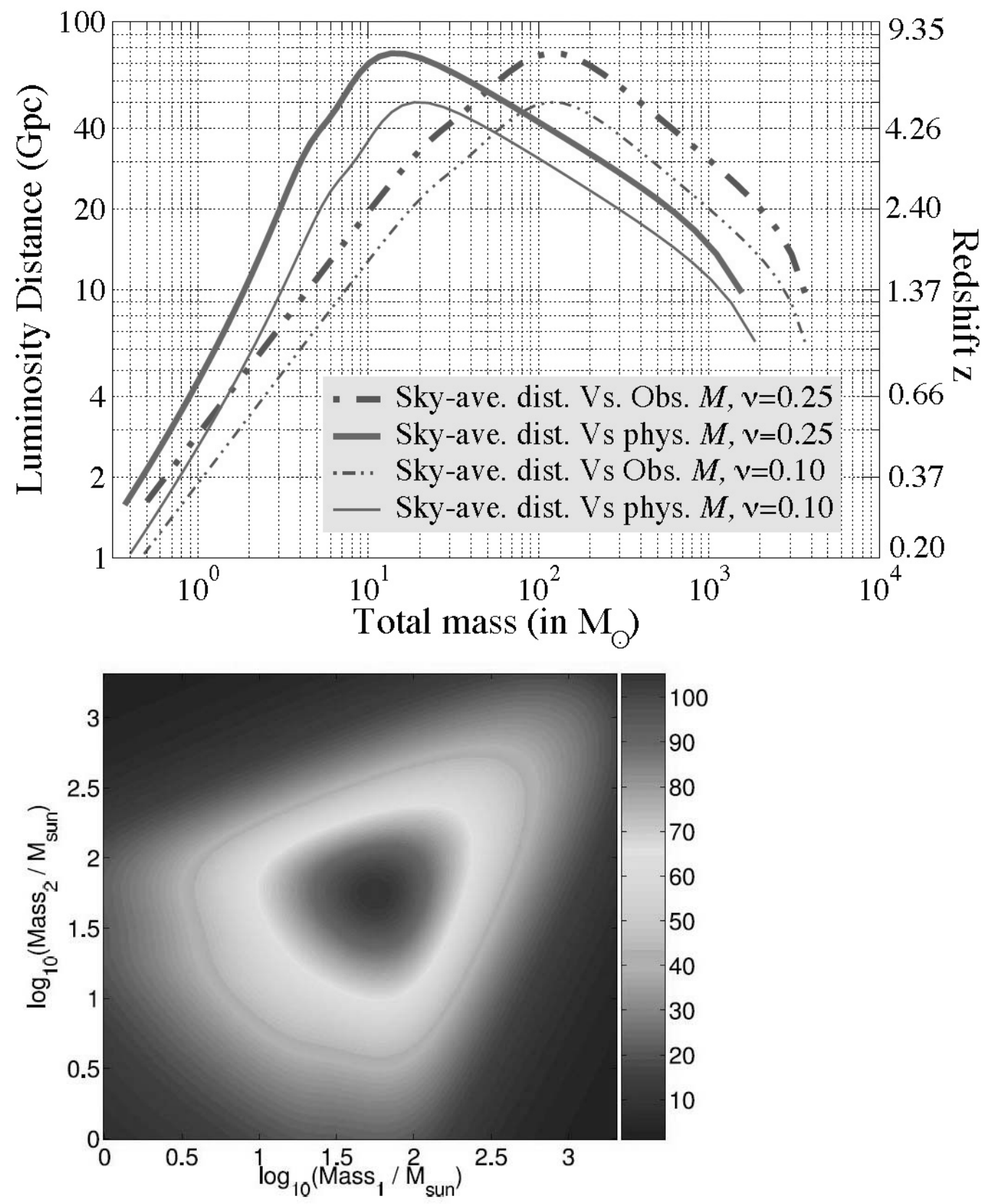

Figure 1.2 Upper panel: The detection distance of ET-B (at an $\mathrm{SNR}=10$ ) for inspiral signals as a function of the intrinsic (red solid line) and observed (blue dashed line) total mass. Lower panel: SNR for binaries at a distance of $3 \mathrm{Gpc}$ as a function of the component masses (Amaro-Seoane et al., 2009; Punturo et al., 2009). 


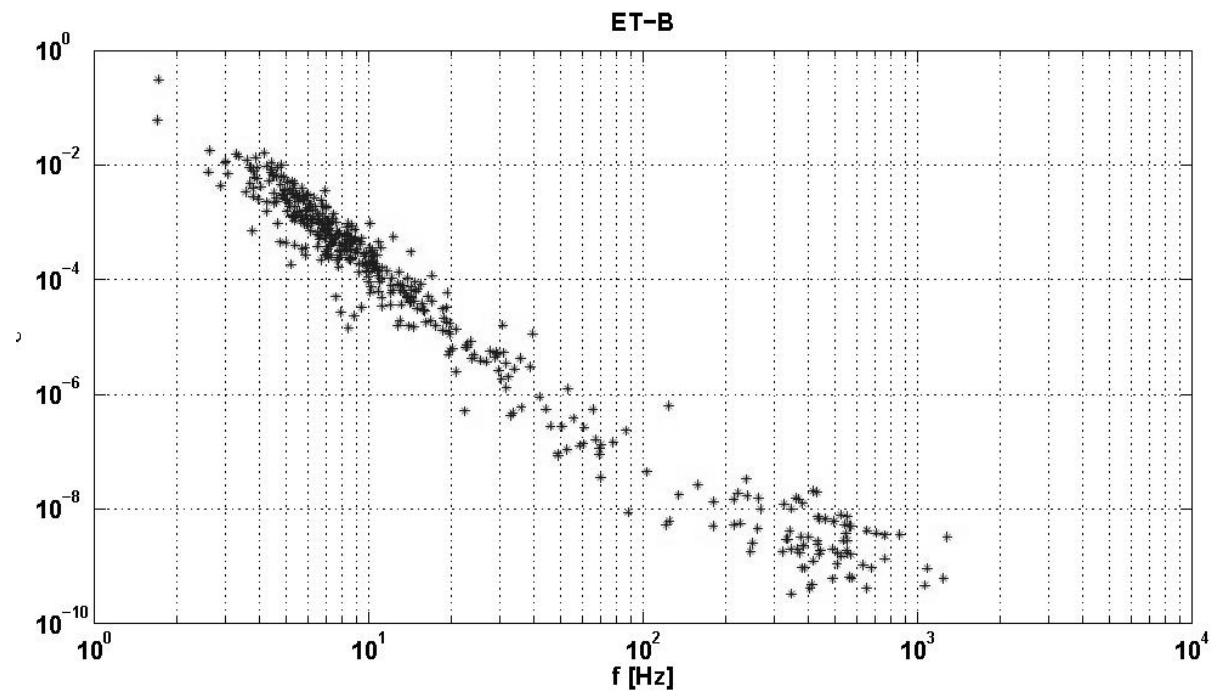

Figure 1.3 Smallest ellipticity of known pulsars detectable in ET (ET-B sensitivity) Amaro-Seoane et al. (2009).

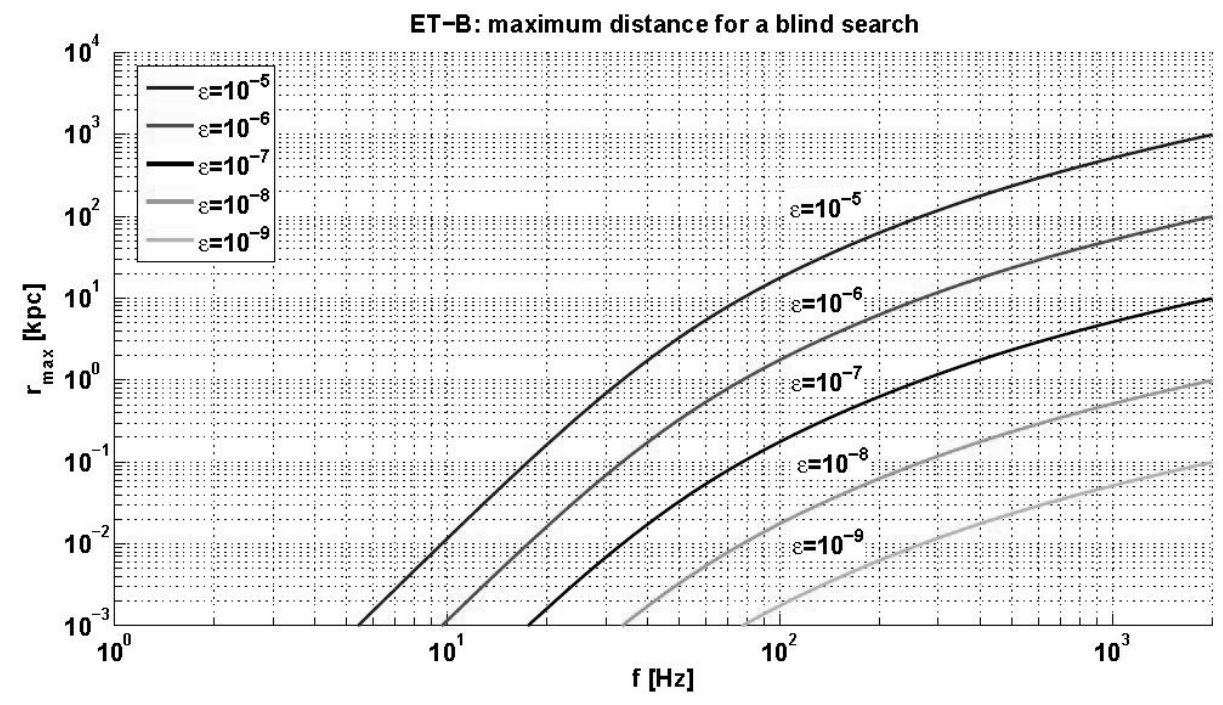

Figure 1.4 The detection range for a blind search of NS in ET (ET-B sensitivity) (Amaro-Seoane et al., 2009). 


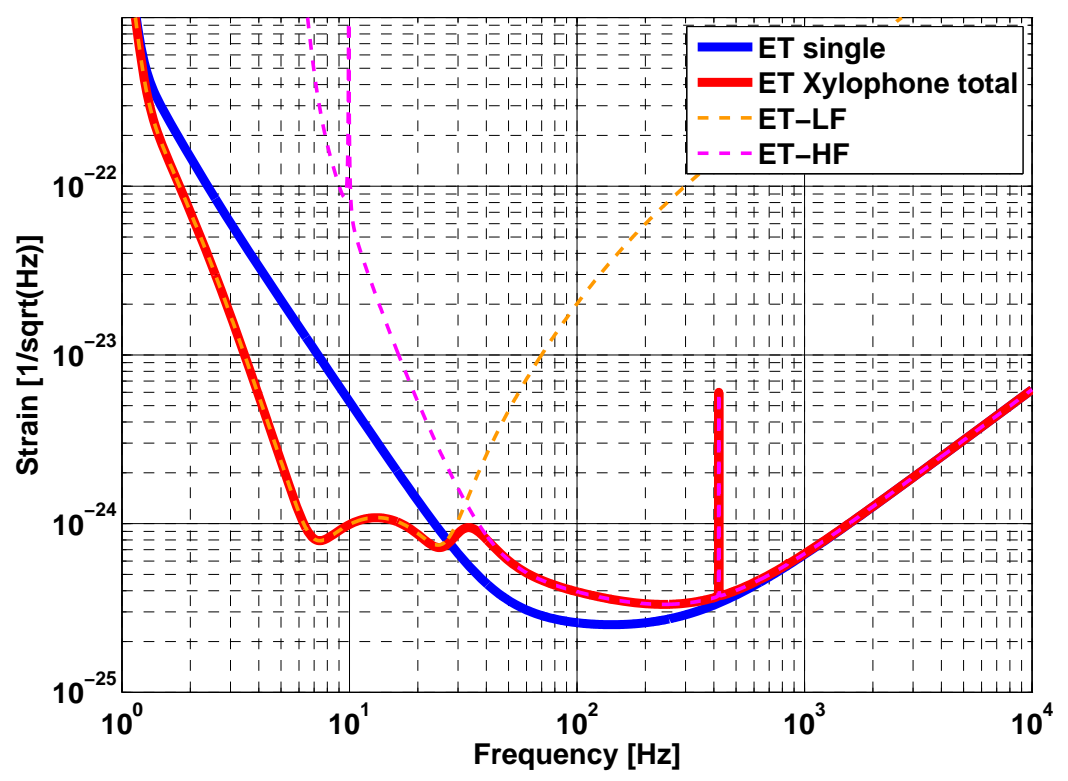

Figure 1.5 Sensitivity of a third generation GW observatory implemented by two frequency-specialised (LF \& HF) detectors (Xylophone topology (Hild et al., 2010), curve so-called "ET-C"), with respect to a single wide frequency range interferometer ET implementation 


\section{References}

Abbott, R, Adhikari, R, Allen, G, Cowley, S, Daw, E, DeBra, D, Giaime, J, Hammond, G, Hammond, M, Hardham, C, How, J, Hua, W, Johnson, W, Lantz, B, Mason, K, Mittleman, R, Nichol, J, Richman, S, Rollins, J, Shoemaker, D, Stapfer, G, and Stebbins, R. 2002. Seismic isolation for Advanced LIGO. Classical and Quantum Gravity, 19, 1591-1597.

Acernese, F, Alshourbagy, M, Amico, P, Antonucci, F, Aoudia, S, Astone, P, Avino, S, Baggio, L, Ballardin, G, Barone, F, Barsotti, L, Barsuglia, M, Bauer, Th S, Bigotta, S, Birindelli, S, Bizouard, M A, Boccara, C, Bondu, F, Bosi, L, Braccini, S, Bradaschia, C, Brillet, A, Brisson, V, Buskulic, D, Cagnoli, G, Calloni, E, Campagna, E, Carbognani, F, Cavalier, F, Cavalieri, R, Cella, G, Cesarini, E, Chassande-Mottin, E, Clapson, A-C, Cleva, F, Coccia, E, Corda, C, Corsi, A, Cottone, F, Coulon, J-P, Cuoco, E, D'Antonio, S, Dari, A, Dattilo, V, Davier, M, Rosa, R De, Prete, M Del, Fiore, L Di, Lieto, A Di, Emilio, M Di Paolo, Virgilio, A Di, Evans, M, Fafone, V, Ferrante, I, Fidecaro, F, Fiori, I, Flaminio, R, Fournier, J-D, Frasca, S, Frasconi, F, Gammaitoni, L, Garufi, F, Genin, E, Gennai, A, Giazotto, A, Giordano, L, Granata, V, Greverie, C, Grosjean, D, Guidi, G, Hamdani, S, Hebri, S, Heitmann, H, Hello, P, Huet, D, Kreckelbergh, S, Penna, P La, Laval, M, Leroy, N, Letendre, N, Lopez, B, Lorenzini, M, Loriette, V, Losurdo, G, Mackowski, J-M, Majorana, E, Man, C N, Mantovani, M, Marchesoni, F, Marion, F, Marque, J, Martelli, F, Masserot, A, Menzinger, F, Milano, L, Minenkov, Y, Moins, C, Moreau, J, Morgado, N, Mosca, S, Mours, B, Neri, I, Nocera, F, Pagliaroli, G, Palomba, C, Paoletti, F, Pardi, S, Pasqualetti, A, Passaquieti, R, Passuello, D, Piergiovanni, F, Pinard, L, Poggiani, R, Punturo, M, Puppo, P, Rapagnani, P, Regimbau, T, Remillieux, A, Ricci, F, Ricciardi, I, Rocchi, A, Rolland, L, Romano, R, Ruggi, P, Russo, G, Solimeno, S, Spallicci, A, Tarallo, M, Terenzi, R, Toncelli, A, Tonelli, M, Tournefier, E, Travasso, F, Tremola, C, Vajente, G, van den Brand, J F J, van der Putten, S, Verkindt, D, Vetrano, F, Vicere, A, Vinet, J-Y, Vocca, H, and Yvert, M. 2008. Status of Virgo. Classical and Quantum Gravity, 25(11), 114045 (8pp).

Agresti, Juri, Castaldi, Giuseppe, DeSalvo, Riccardo, Galdi, Vincenzo, Pierro, Vincenzo, and Pinto, Innocenzo M. 2006. Optimized multilayer dielectric 
mirror coatings for gravitational wave interferometers. Advances in ThinFilm Coatings for Optical Applications III, 6286(1), 628608.

Ajith, P., Babak, S., Chen, Y., Hewitson, M., Krishnan, B., Sintes, A. M., Whelan, J. T., Brügmann, B., Diener, P., Dorband, N., Gonzalez, J., Hannam, M., Husa, S., Pollney, D., Rezzolla, L., Santamaría, L., Sperhake, U., and Thornburg, J. 2008. Template bank for gravitational waveforms from coalescing binary black holes: Nonspinning binaries. Phys. Rev. D, 77(10), 104017.

Alshourbagy, M., Amico, P., Bosi, L., Cagnoli, G., Campagna, E., Cottone, F., Dari, A., Gammaitoni, L., Lorenzini, M., Losurdo, G., Marchesoni, F., Martelli, F., Piergiovanni, F., Punturo, M., Toncelli, A., Tonelli, M., Travasso, F., Vetrano, F., and Vocca, H. 2006. First characterization of silicon crystalline fibers produced with the mu-pulling technique for future gravitational wave detectors. Review of Scientific Instruments, $\mathbf{7 7}(4), 044502$.

Amaro-Seoane, Pau, Andersson, Nils, Arun, K.G., Bose, Sukanta, Bosi, Leone, Bulik, Tomasz, Clark, James, Dent, Thomas, Gair, Jonathan, Glampedakis, Kostas, Hannam, Mark, Heng, Siong, Husa, Sascha, Mandel, Ilya, Jones, Gareth, Krishnan, Badri, Mandel, Ilya, O'Shaughnessy, Richard, Ott, Christian, Read, Jocelyn, Regimbau, Tania, Rezzolla, Luciano, Sathyaprakash, B.S., Broeck, Chris Van Den, Vecchio, Alberto, and Veitch, John. 2009. Einstein Telescope Design Study: Vision Document, https://workarea.et-gw.eu/et/WG4-Astrophysics/visdoc/. ET internal note: ET-031-09.

Amico, P., Bosi, L., Carbone, L., Gammaitoni, L., Marchesoni, F., Punturo, M., Travasso, F., and Vocca, H. 2002. Monolithic fused silica suspension for the Virgo gravitational waves detector. Review of Scientific Instruments, 73(9), 3318-3323.

Ando, Shin'ichiro, Beacom, John F., and Yüksel, Hasan. 2005. Detection of Neutrinos from Supernovae in Nearby Galaxies. Phys. Rev. Lett., 95(17), 171101.

Arnaud, Nicolas, Barsuglia, Matteo, Bizouard, Marie-Anne, Canitrot, Philippe, Cavalier, Fabien, Davier, Michel, Hello, Patrice, and Pradier, Thierry. 2002. Detection in coincidence of gravitational wave bursts with a network of interferometric detectors: Geometric acceptance and timing. Physical Review D (Particles, Fields, Gravitation, and Cosmology) Rev. $D, 65(4), 042004$.

Arun, K. G., Iyer, Bala R., Sathyaprakash, B. S., and Sinha, Siddhartha. 2007. Higher harmonics increase LISA?s mass reach for supermassive black holes. Physical Review D (Particles, Fields, Gravitation, and Cosmology), 75, 124002.

Baker, John G., van Meter, James R., McWilliams, Sean T., Centrella, Joan, and Kelly, Bernard J. 2007. Consistency of Post-Newtonian Waveforms with Numerical Relativity. Phys. Rev. Lett., 99(18), 181101.

Beccaria, M, et al. 1998. Relevance of Newtonian seismic noise for the VIRGO interferometer sensitivity. Classical and Quantum Gravity, 15, 33393362 . 
Beker, M G, DeSalvo, R, Doets, M, Dorsher, S, Hennes, E, Grote, H, Harms, J, Kuroda, K, Mandic, V, Rabeling, D S, van den Brand, J F J, and van Leeuwen, C M. 2009. Selection criteria for ET candidate site. ER internal note: ET-030-09.

Bergh, S V, and Tammann, G A. 1991. Galactic and Extragalactic Supernova Rates. Annual Review of Astronomy and Astrophysics, 29, 363-407.

Boyle, Michael, Brown, Duncan A., Kidder, Lawrence E., Mroué, Abdul H., Pfeiffer, Harald P., Scheel, Mark A., Cook, Gregory B., and Teukolsky, Saul A. 2007. High-accuracy comparison of numerical relativity simulations with post-Newtonian expansions. Phys. Rev. D, 76(12), 124038.

Braccini, S:. 2009. Superattenuator seismic isolation measurements by Virgo interferometer: a comparison with the future generation antenna. ET internal note ET-025-09.

Braccini, S., Barsotti, L., Bradaschia, C., Cella, G., Virgilio, A. Di, Ferrante, I., Fidecaro, F., Fiori, I., Frasconi, F., Gennai, A., Giazotto, A., Paoletti, F., Passaquieti, R., Passuello, D., Poggiani, R., Campagna, E., Guidi, G., Losurdo, G., Martelli, F., Mazzoni, M., Perniola, B., Piergiovanni, F., Stanga, R., Vetrano, F., Vicer?, A., Brocco, L., Frasca, S., Majorana, E., Pai, A., Palomba, C., Puppo, P., Rapagnani, P., Ricci, F., Ballardin, G., Barill?, R., Cavalieri, R., Cuoco, E., Dattilo, V., Enard, D., Flaminio, R., Freise, A., Hebri, S., Holloway, L., Penna, P. La, Loupias, M., Marque, J., Moins, C., Pasqualetti, A., Ruggi, P., Taddei, R., Zhang, Z., Acernese, F., Avino, S., Barone, F., Calloni, E., Rosa, R. De, Fiore, L. Di, Eleuteri, A., Giordano, L., Milano, L., Pardi, S., Qipiani, K., Ricciardi, I., Russo, G., Solimeno, S., Babusci, D., Giordano, G., Amico, P., Bosi, L., Gammaitoni, L., Marchesoni, F., Punturo, M., Travasso, F., Vocca, H., Boccara, C., Moreau, J., Loriette, V., Reita, V., Mackowski, J.M., Morgado, N., Pinard, L., Remillieux, A., Barsuglia, M., Bizouard, M.A., Brisson, V., Cavalier, F., Clapson, A.C., Davier, M., Hello, P., Krecklbergh, S., Beauville, F., Buskulic, D., Gouaty, R., Grosjean, D., Marion, F., Masserot, A., Mours, B., Tournefier, E., Tombolato, D., Verkindt, D., Yvert, M., Aoudia, S., Bondu, F., Brillet, A., Chassande-Mottin, E., Cleva, F., Coulon, J.P., Dujardin, B., Fournier, J.D., Heitmann, H., Man, C.N., Spallicci, A., and Vinet, J.Y. 2005. Measurement of the seismic attenuation performance of the VIRGO Superattenuator. Astroparticle Physics, 23(6), 557 - 565.

Braginsky, V. B., Strigin, S. E., and Vyatchanin, S. P. 2001. Parametric oscillatory instability in Fabry-Perot interferometer. Physics Letters A, 287(5-6), $331-338$.

Broeck, Chris Van Den. 2006. Binary black hole detection rates in inspiral gravitational wave searches. Classical and Quantum Gravity, 23, L51L58.

Broeck, Chris Van Den, and Sengupta, Anand S. 2007. Phenomenology of amplitude-corrected post-Newtonian gravitational waveforms for compact binary inspiral: I. Signal-to-noise ratios. Classical and Quantum Gravity, 24, 155-176.

Brückner, Frank, Friedrich, Daniel, Clausnitzer, Tina, Burmeister, Oliver, 
Britzger, Michael, Kley, Ernst-Bernhard, Danzmann, Karsten, Tünnermann, Andreas, and Schnabel, Roman. 2009. Demonstration of a cavity coupler based on a resonant waveguide grating. Opt. Express, 17(1), 163-169.

Brügmann, Bernd, Tichy, Wolfgang, and Jansen, Nina. 2004. Numerical Simulation of Orbiting Black Holes. Phys. Rev. Lett, 92, 211101.

Bunkowski, A, Burmeister, O, Clausnitzer, T, Kley, E-B, Tnnermann, A, Danzmann, K, and Schnabel, R. 2006. Diffractive Optics for Gravitational Wave Detectors. Journal of Physics: Conference Series, 32(1), 333 .

Buonanno, A., and Damour, T. 1999. Effective one-body approach to general relativistic two-body dynamics. Phys.Rev. D, 59, 084006.

Buonanno, Alessandra, and Damour, Thibault. 2000. Transition from inspiral to plunge in binary black hole coalescences. Phys.Rev. D, 62, 064015.

Buonanno, Alessandra, Pan, Yi, Baker, John G., Centrella, Joan, Kelly, Bernard J., McWilliams, Sean T., and van Meter, James R. 2007. Toward faithful templates for non-spinning binary black holes using the effective-one-body approach. Phys.Rev.D, 76:104049,2007(June).

Cagnoli, G., Gammaitoni, L., Kovalik, J., Marchesoni, F., and Punturo, M. 1996. Suspension losses in low-frequency mechanical pendulums. Physics Letters A, 213(5-6), 245 - 252.

Cagnoli, G., Gammaitoni, L., Kovalik, J., Marchesoni, F., and Punturo, M. 1999. Low-frequency internal friction in clamped-free thin wires. Physics Letters A, 255(4-6), 230 - 235.

Cagnoli, G, et al. 2006. Silica suspension and coating developments for Advanced LIGO. Journal of Physics Conference Series, 32, 386.

Callen, Herbert B., and Welton, Theodore A. 1951. Irreversibility and Generalized Noise. Phys. Rev., 83(1), 34-40.

Cella, G. 2009. Gravity Gradient Noise: estimates \& reduction strategies. Talk at the 2nd ET Annual meeting, http://www.etgw.eu/2ndgeneralworkshop.

Chelkowski, Simon, Hild, Stefan, and Freise, Andreas. 2009. Prospects of higher-order Laguerre-Gauss modes in future gravitational wave detectors. Phys. Rev. D, 79(12), 122002.

Conforto, Gianni, and DeSalvo, Riccardo. 2004. Proposal for lower frequency companions for the advanced LIGO Gravitational Wave Interferometric Detectors. Nuclear Instruments and Methods in Physics Research Section A: Accelerators, Spectrometers, Detectors and Associated Equipment, 518(1-2), 228 - 232. Frontier Detectors for Frontier Physics: Proceedin.

Damour, Thibault, and Nagar, Alessandro. 2007a. Comparing Effective-OneBody gravitational waveforms to accurate numerical data. Phys.Rev.D, 77:024043,2008(Nov.).

Damour, Thibault, and Nagar, Alessandro. 2007b. Faithful EffectiveOne-Body waveforms of small-mass-ratio coalescing black-hole binaries. Phys.Rev.D, 76:064028,2007(May). 
Damour, Thibault, Iyer, Bala R., and Sathyaprakash, B. S. 2002. A comparison of search templates for gravitational waves from binary inspiral - 3.5PN update. Phys.Rev. D, 66, 027502.

Damour, Thibault, Iyer, Bala R., Jaranowski, Piotr, and Sathyaprakash, B. S. 2003. Gravitational waves from black hole binary inspiral and merger: The span of third post-Newtonian effective-one-body templates. Phys.Rev. D, 67, 064028.

Freise, A, Chelkowski, S, Hild, S, Pozzo, W Del, Perreca, A, and Vecchio, A. 2009. Triple Michelson interferometer for a third-generation gravitational wave detector. Classical and Quantum Gravity, 26(8), 085012 (14pp).

Gair, Jonathan R., Mandel, Ilya, Miller, M. Coleman, and Volonteri, Marta. 2009a. Exploring intermediate and massive black-hole binaries with the Einstein Telescope. arXiv:090\%.5450, July.

Gair, Jonathan R, Mandel, Ilya, Sesana, Alberto, and Vecchio, Alberto. 2009b. Probing seed black holes using future gravitational-wave detectors. Classical and Quantum Gravity, 26(July), 204009.

Goßler, S, Cagnoli, G, Crooks, D R M, Lück, H, Rowan, S, Smith, J R, Strain, K A, Hough, J, and Danzmann, K. 2004. Damping and tuning of the fibre violin modes in monolithic silica suspensions. Classical and Quantum Gravity, 21, S923.

Green, Martin A., and Keevers, Mark J. 1995. Optical properties of intrinsic silicon at $300 \mathrm{~K}$. Progress in Photovoltaics: Research and Applications, 3, 189-192.

Hannam, Mark, and Hawke, Ian. 2009. Numerical relativity simulations in the era of the Einstein Telescope. arXiv:0908.3139v1 [gr-qc], Aug.

Harms, Jan, Chen, Yanbei, Chelkowski, Simon, Franzen, Alexander, Vahlbruch, Henning, Danzmann, Karsten, and Schnabel, Roman. 2003. Squeezed-input, optical-spring, signal-recycled gravitational-wave detectors. Phys. Rev. D, 68(4), 042001.

Harry, Gregory M, Abernathy, Matthew R, Becerra-Toledo, Andres E, Armandula, Helena, Black, Eric, Dooley, Kate, Eichenfield, Matt, Nwabugwu, Chinyere, Villar, Akira, Crooks, D R M, Cagnoli, Gianpietro, Hough, Jim, How, Colin R, MacLaren, Ian, Murray, Peter, Reid, Stuart, Rowan, Sheila, Sneddon, Peter H, Fejer, Martin M, Route, Roger, Penn, Steven D, Ganau, Patrick, Mackowski, Jean-Marie, Michel, Christophe, Pinard, Laurent, and Remillieux, Alban. 2007. Titania-doped tantala/silica coatings for gravitational-wave detection. Classical and Quantum Gravity, $\mathbf{2 4}(2), 405-415$.

Hild, S, Chelkowski, S, Freise, A, Franc, J, Morgado, N, Flaminio, R, and DeSalvo, R. 2010. A xylophone configuration for a third-generation gravitational wave detector. Classical and Quantum Gravity, 27(1), 015003 (8pp).

Hild, Stefan. 2009. Khalili-Cavities for ET? Talk at the ET-WP3 meeting in Glasgow (December 2009).

Hild, Stefan, Chelkowski, Simon, and Freise, Andreas. 2008. Pushing towards the ET sensitivity using 'conventional' technology. arXiv:0810.0604v2 $[g r-q c]$. 
http://www.et-gw.eu/. 2009. The Einstein Telescope design study (FPryCapacities, Grant Agreement 211743).

Hughes, Scott A., and Thorne, Kip S. 1998. Seismic gravity-gradient noise in interferometric gravitational-wave detectors. Phys. Rev. D, 58(12), 122002.

Ju, L., Zhao, C., Gras, S., Degallaix, J., Blair, D.G., Munch, J., and Reitze, D.H. 2006a. Comparison of parametric instabilities for different test mass materials in advanced gravitational wave interferometers. Physics Letters A, 355(6), $419-426$.

Ju, L., Gras, S., Zhao, C., Degallaix, J., and Blair, D.G. 2006b. Multiple modes contributions to parametric instabilities in advanced laser interferometer gravitational wave detectors. Physics Letters A, 354, 360-365.

Khalili, F.Ya. 2005. Reducing the mirrors coating noise in laser gravitationalwave antennae by means of double mirrors. Physics Letters A, 334(1), $67-72$.

Kimble, H. J., Levin, Yuri, Matsko, Andrey B., Thorne, Kip S., and Vyatchanin, Sergey P. 2001. Conversion of conventional gravitational-wave interferometers into quantum nondemolition interferometers by modifying their input and/or output optics. Phys. Rev. D, 65(2), 022002.

Martin, I, et al. 2008. Measurements of a low-temperature mechanical dissipation peak in a single layer of $\mathrm{Ta}_{2} \mathrm{O}_{5}$ doped with $\mathrm{TiO}_{2}$. Classical and Quantum Gravity, 25, 055005.

Meers, Brian J. 1988. Recycling in laser-interferometric gravitational-wave detectors. Phys. Rev. D, 38(8), 2317-2326.

Mehmet, Moritz, Vahlbruch, Henning, Lastzka, Nico, Danzmann, Karsten, and Schnabel, Roman. 2009. Observation of squeezed states with strong photon number oscillations. http://arxiv.org/pdf/0909.5386.

Nakar, Ehud. 2007. Short-hard gamma-ray bursts. Physics Reports, 442, 166-236.

Ott, Christian D. 2009. The gravitational-wave signature of core-collapse supernovae. Classical and Quantum Gravity, 26, 063001.

Pretorius, Frans. 2005. Evolution of Binary Black-Hole Spacetimes. Phys. Rev. Lett., 95, 121101.

Punturo, M, Abernathy, M, Acernese, F, Allen, B, Andersson, N, Arun, K, Barone, F, Barr, B, Barsuglia, M, Beker, M, Beveridge, N, Birindelli, S, Bose, S, Bosi, L, Braccini, S, Bradaschia, C, Bulik, T, Calloni, E, Cella, G, Chassande-Mottin, E, Chelkowski, S, Chincarini, A, Clark, J, Coccia, E, Colacino, C, Cumming, A, Cunningham, L, Cuoco, E, Danilishin, S, Danzmann, K, Luca, G De, Salvo, R De, Dent, T, Derosa, R, Fiore, L Di, Virgilio, A Di, Doets, M, Fafone, V, Falferi, P, Flaminio, R, Franc, J, Frasconi, F, Freise, A, Fulda, P, Gair, J, Gemme, G, Gennai, A, Giazotto, A, Glampedakis, K, Granata, M, Grote, H, Guidi, G, Hammond, G, Hannam, M, Harms, J, Heinert, D, Hendry, M, Heng, I, Hennes, E, Hild, S, Hough, J, Husa, S, Huttner, S, Jones, G, Khalili, F, Kokeyama, K, Kokkotas, K, Krishnan, B, Lorenzini, M, Lueck, H, Majorana, E, Mandel, I, Mandic, V, Martin, I, Michel, C, Minenkov, Y, Morgado, N, Mosca, 
S, Mours, B, Mueller-Ebhardt, H, Murray, P, Nawrodt, R, Nelson, J, Oshaughnessy, R, Ott, C D, Palomba, C, Paoli, A, Parguez, G, Pasqualetti, A, Passaquieti, R, Passuello, D, Pinard, L, Poggiani, R, Popolizio, P, Prato, M, Puppo, P, Rabeling, D, Rapagnani, P, Read, J, Regimbau, T, Rehbein, H, Reid, S, Rezzolla, L, Ricci, F, Richard, F, Rocchi, A, Rowan, S, Ruediger, A, Sassolas, B, Sathyaprakash, B, Schnabel, R, Schwarz, C, Seidel, P, Sintes, A, Somiya, K, Speirits, F, Strain, K, Strigin, S, Sutton, P, Tarabrin, S, van den Brand, J, van Leewen, C, van Veggel, M, van den Broeck, C, Vecchio, A, Veitch, J, Vetrano, F, Vicere, A, Vyatchanin, S, Willke, B, Woan, G, Wolfango, P, and Yamamoto, K. 2009. Third Generation of Gravitational Wave Observatories and their Science Reach. submitted to Classical and Quantum Gravity.

Reid, S., Cagnoli, G., Crooks, D.R.M., Hough, J., Murray, P., Rowan, S., Fejer, M.M., Route, R., and Zappe, S. 2006. Mechanical dissipation in silicon flexures. Physics Letters A, 351(4-5), $205-211$.

Sathyaprakash, B. S., Schutz, Bernard, and Broeck, Chris Van Den. 2009. Cosmography with the Einstein Telescope. arXiv:0906.4151v1 [astroph. CO], June.

Sato, Shuichi, Miyoki, Shinji, Telada, Souichi, Tatsumi, Daisuke, Araya, Akito, Ohashi, Masatake, Totsuka, Yoji, Fukushima, Mitsuhiro, and Fujimoto, Masa-Katsu. 2004. Ultrastable performance of an underground-based laser interferometer observatory for gravitational waves. Phys. Rev. D, 69(10), 102005.

Saulson, Peter R. 1984. Terrestrial gravitational noise on a gravitational wave antenna. Phys. Rev. D, 30(4), 732-736.

Schutz, Bernard F. 1986. Determining the Hubble constant from gravitational wave observations. Nature, 323, 310-311.

Shoemaker, David. 2001. Future limits to sensitivity. G010026-00-G.

Tomaru, T., Suzuki, T., Uchiyama, T., Yamamoto, A., Shintomi, T., Taylor, C. T., Yamamoto, K., Miyoki, S., Ohashi, M., and Kuroda, K. 2002. Maximum heat transfer along a sapphire suspension fiber for acryogenic interferometric gravitational wave detector. Physics Letters A, 301(3-4), $215-219$.

Tomaru, Takayuki, Uchiyama, Takashi, Tatsumi, Daisuke, Miyoki, Shinji, Ohashi, Masatake, Kuroda, Kazuaki, Suzuki, Toshikazu, Yamamoto, Akira, and Shintomi, Takakazu. 2001. Cryogenic measurement of the optical absorption coefficient in sapphire crystals at $1.064[\mathrm{mu}] \mathrm{m}$ for the large-scale cryogenic gravitational wave telescope. Physics Letters A, $\mathbf{2 8 3}(1-2), 80-84$.

Tomaru, Takayuki, et al. 2002. Thermal lensing in cryogenic sapphire substrates. Classical and Quantum Gravity, 19, 2045.

Tomaru, Takayuki, et al. 2004. Development of a cryocooler vibrationreduction system for a cryogenic interferometric gravitational wave detector. Classical and Quantum Gravity, 21, S1005.

Uchiyama, T., Tomaru, T., Tatsumi, D., Miyoki, S., Ohashi, M., Kuroda, K., Suzuki, T., Yamamoto, A., and Shintomi, T. 2000. Mechanical quality 
factor of a sapphire fiber at cryogenic temperatures. Physics Letters A, 273(5-6), $310-315$.

Vahlbruch, Henning, Chelkowski, Simon, Hage, Boris, Franzen, Alexander, Danzmann, Karsten, and Schnabel, Roman. 2006. Coherent Control of Vacuum Squeezing in the Gravitational-Wave Detection Band. Phys. Rev. Lett., 97(1), 011101.

Vahlbruch, Henning, Mehmet, Moritz, Chelkowski, Simon, Hage, Boris, Franzen, Alexander, Lastzka, Nico, Goßler, Stefan, Danzmann, Karsten, and Schnabel, Roman. 2008. Observation of Squeezed Light with 10-dB Quantum-Noise Reduction. Phys. Rev. Lett., 100(3), 033602.

Waldman for the LIGO Science Collaboration, Samuel J. 2006. Status of LIGO at the start of the fifth science run. Classical and Quantum Gravity, 23(19), S653-S660.

Winkler, W., Maischberger, K., Ruediger, A., Schilling, R., Schnupp, L., Hoemaker, D., and D. Shoemaker, W. Winkler, K. Maischberger, A. Ruediger, R. Schilling, \& L. Schnupp (eds). 1985 (Aug.). Plans for a large gravitational wave antenna in Germany.

Yamamoto, K, Uchiyama, T, Miyoki, S, Ohashi, M, Kuroda, K, and Numata, K. 2008. Parametric instabilities in the LCGT arm cavity. Journal of Physics: Conference Series, 122, 012015 (6pp).

Yamamoto, Kazuhiro, Miyoki, Shinji, Uchiyama, Takashi, Ishitsuka, Hideki, Ohashi, Masatake, Kuroda, Kazuaki, Tomaru, Takayuki, Sato, Nobuaki, Suzuki, Toshikazu, Haruyama, Tomiyoshi, Yamamoto, Akira, Shintomi, Takakazu, Numata, Kenji, Waseda, Koichi, Ito, Kazuhiko, and Watanabe, Koji. 2006. Measurement of the mechanical loss of a cooled reflective coating for gravitational wave detection. Phys. Rev. D, 74(2), 022002. 